\title{
Papel de la clorhexidina en la odontología restauradora
}

Role of chlorhexidine in restorative dentistry

Artículo DE REvisión

\section{Resumen}

La durabilidad de la unión de los materiales restauradores al tejido dentario es un importante requisito para conseguir tratamientos exitosos en la Odontología. Desafortunadamente, la capa híbrida formada entre la resina y la dentina sufre procesos degradativos, tanto del componente resinoso como del colágeno. La clorhexidina es un agente antibacteriano de amplio espectro con capacidad de inhibir la actividad proteolítica de algunas enzimas que pueden degradar las fibras colágenas de la capa híbrida, razón por la cual fue recientemente incorporada al protocolo de aplicación de adhesivos dentales convencionales. El presente artículo revisa estudios en la literatura científica acerca del uso de clorhexidina en la Odontología Restauradora para aumentar la longevidad de las uniones resina-dentina.

Palabras clave: Adhesivos dentales, clorhexidina, longevidad, durabilidad, uniones resina-dentina, metaloproteinasas de la matriz (MMPs).

\section{Abstract}

The durability of the union of the restorative materials to the dental tissue is an important requirement to obtain successful treatments in Dentistry. Unfortunately, the hybrid layer formed between the resin and the dentine undergoes degradative processes, of both the resin component such as the collagen. The chlorhexidine is a wide-spectrum antibacterial agent with inhibition capability against the proteolytic activity of some enzymes that can degrade the collagen fibers from the hybrid layer. For that reason, it was recently incorporated to the conventional dental adhesive application protocol. This article reviews studies on scientific literature about the use of chlorhexidine in Restorative Dentistry to increase resindentine unions' longevity.

Key words: Dental adhesives, chlorhexidine, longevity, durability, resin-dentine unions, matrix metalloproteinases (MMPs).

\section{César Pomacóndor-Hernández}

\author{
Maestrando en Materiales Dentales. Facultad de \\ Odontología de Piracicaba, UNICAMP. Sao Pau- \\ lo - Brasil.
}

\section{Correspondencia:}

CD César Pomacóndor-Hernández

Faculdade de Odontologia de Piracicaba, UNICAMP. Departamento de Odontologia Restauradora. Área de Materiais Dentários. Av. Limeira 901, Caixa Postal 52, CEP 13414-903. Piracicaba - SP, Brasil. Telefone: +55 1921065345.

E-mail: cesar.pomacondor.hernandez@gmail.com

Fecha de recepción: 10-07-10

Fecha de aprobación: 08-11-10

\section{Introducción}

A partir de la segunda mitad del Siglo $\mathrm{XX}$, la creciente demanda biológica de conservación de estructura dentaria en los procedimientos restauradores trajo consigo el desarrollo de los sistemas adhesivos. ${ }^{1}$ Con esto se facilitó satisfacer otras dos condiciones no menos importantes: la estética de las restauraciones dentales $^{2}$ y la compatibilidad biomecánica entre el material restaurador y el diente. ${ }^{3}$ Tras varios ańos de evolución de los sistemas adhesivos, clasificados al inicio en generaciones, ${ }^{4}$ fue propuesta una nueva clasificación de las estrategias de unión a la estructura dentaria que consiste en: adhesivos convencionales ("de grabar y lavar"), adhesivos autograbadores, y materiales autoadhesivos. ${ }^{5,6}$

La estrategia de unión de los adhesivos convencionales consiste en la desmineralización de la dentina con un ácido para exponer las fibras colágenas, seguido por la infiltración de monómeros resinosos sobre estas fibras expuestas (primer + adhesivo, o solamente adhesivo), y finalmente la polimerización in situ de estos monómeros para formar la denominada capa híbrida. ${ }^{7}$ Desafor- tunadamente los monómeros resinosos no llegan a infiltrar completamente las fibras colágenas expuestas resultando en una zona de dentina desmineralizada debajo de la capa híbrida, ${ }^{8}$ propiciando que las uniones resina-dentina presenten degradación con el tiempo, ${ }^{9}$ tanto por hidrólisis del adhesivo como de las fibras colágenas. ${ }^{10}$

Las fibras colágenas no protegidas de las regiones adhesivas pueden ser degradadas por enzimas proteolíticas endógenas que se encuentran en la dentina llamadas metaloproteinasas de la matriz (MMPs). ${ }^{11}$ Han sido identificadas 4 tipos de MMPs en la dentina: MMP-2 y MMP-9 (gelatinasas), MMP-8 (colagenasa), y MMP-20 (enamelisina). ${ }^{12}$ Estas enzimas son responsables de la degradación de la matriz extracelular en diferentes procesos fisiológicos (morfogénesis de los dientes) y patológicos (caries dental). ${ }^{12}$

La clorhexidina es un compuesto de propiedades antibacterianas ${ }^{13}$ y a su vez es un inhibidor de la actividad proteolítica de las MMPs. ${ }^{14} \mathrm{Al}$ ser aplicada sobre la dentina en un procedimiento adhesivo puede inhibir la degradación de las fibras colágenas de la capa híbrida contribuyendo a una mayor longevidad de la unión resina-dentina. ${ }^{15}$

\section{Objetivos}

El propósito de este artículo fue revisar estudios en la literatura científica acerca del uso de clorhexidina en la Odontología Restauradora para aumentar la longevidad de las uniones resina-dentina.

\section{Revisión de la literatura}

\section{Clorhexidina y odontología: estudios iniciales}

La clorhexidina es un compuesto químico sintético descubierto durante una investigación sobre las propiedades biológicas de algunas polibiguanidas y seleccionada entre todas como la que presentaba mayor actividad antibacteriana. ${ }^{13}$ Desde entonces, debido al amplio espectro antibacteriano que posee, la clorhexidina ha sido utilizada en el área de las ciencias médicas como agente antiséptico en diversas situaciones clínicas como limpieza de las manos, preparación prequirúrgica de la piel, 
colocación de catéteres y en la higiene oral. $^{16}$

En la Odontología Restauradora, la clorhexidina fue inicialmente introducida como desinfectante de las cavidades antes de la aplicación de los materiales restauradores con el objetivo de eliminar las bacterias que podían irritar la pulpa produciendo sensibilidad postoperatoria y caries secundaria. ${ }^{17}$ Mientras algunos trabajos demostraban incompatibilidad entre la aplicación de clorhexidina a la estructura dentaria y la adhesión de materiales resinosos, ${ }^{18-20}$ otros estudios probaron y confirmaron que la clorhexidina no afectaba la resistencia de unión inmediata. ${ }^{21-23}$ Esta discrepancia de resultados puede ser explicada por las limitaciones de los ensayos comúnmente aplicados en esa época para evaluar la eficacia de la adhesión. ${ }^{24}$ Sin embargo, dicho protocolo fue posteriormente considerado desnecesario debido a que el ácido fosfórico y los mismos adhesivos dentales pueden presentar actividad antibacteriana. ${ }^{25}$

En 1999, una nueva propiedad de la clorhexidina fue descubierta por Gendron y col., ${ }^{14}$ quienes demostraron que soluciones de clorhexidina pueden inhibir la actividad proteolítica de las MMPs -2, -8 y -9. Estas MMPs juegan un importante papel en las enfermedades inflamatorias destructoras de tejidos como la periodontitis, y con este trabajo fue mejor comprendido el efecto benéfico de la clorhexidina en el tratamiento de esta enfermedad. Paralelamente, Tjäderhane y col. ${ }^{26}$ descubrieron que las MMPs de la dentina son activadas por los ácidos producidos por las bacterias cariogénicas, y son estas enzimas proteolíticas las que participan de la destrucción de la matriz colágena en los procesos cariosos.

Con estos antecedentes y con la evidencia morfológica de que las uniones resina-dentina presentaban degradación de las fibras colágenas con el tiempo, ${ }^{9}$ Pashley y col. ${ }^{11}$ realizaron un estudio en el que, usando matrices de dentina desmineralizada y almacenándolas en saliva artificial durante 250 días, concluyeron que la dentina sana tiene la capacidad de degradar las fibras colágenas desprotegidas (libres de hidroxiapatita debido al grabado ácido) en ausencia de colonización bacteriana a través de la acción de las MMPs que son liberadas lentamente a lo largo del tiempo, y la clorhexidina puede actuar como inhibidor de las MMPs en la dentina evitando o retardando este proceso de degradación.
La clorhexidina en el protocolo adhesivo: estudios in vivo

El paso siguiente fue comprobar la efectividad de la clorhexidina en la reducción de la degradación del componente colágeno de la capa híbrida, y considerando que ya existía la corriente de aplicar clorhexidina en el protocolo adhesivo con fines antibacterianos, las investigaciones no pasaron por la fase in vitro $\mathrm{y}$ fueron investigadas directamente in vivo. Hebling y col. ${ }^{15}$ realizaron un estudio en el que incorporaron clorhexidina al protocolo de aplicación de un adhesivo convencional (Single Bond, 3M ESPE) con el objetivo de evitar o disminuir la degradación de la capa híbrida, específicamente de la matriz colágena. En este estudio in vivo fueron usados dientes deciduos cariados contralaterales, y después de la remoción del tejido con un detector de caries, el adhesivo fue aplicado en un lado según las instrucciones del fabricante, $\mathrm{y}$ en el otro fue aplicada una solución de digluconato de clorhexidina al $2 \%$ después del grabado ácido y antes de la aplicación del adhesivo. Después de seis meses de función intraoral, los dientes fueron extraídos y fue demostrado en Microscopía Electrónica de Transmisión (MET) que los dientes tratados con clorhexidina exhibían una región adhesiva con integridad estructural normal de la red colágena, mientras que los dientes no tratados con clorhexidina presentaron desintegración progresiva de la red de fibras colágenas, hasta el punto que no fueron detectadas por la coloración para colágeno. Con este estudio pionero, Hebling y col. ${ }^{15}$ introdujeron un nuevo protocolo de procedimiento adhesivo para prevenir la degradación del colágeno de la capa híbrida. Posteriormente, otra investigación in vivo similar fue realizada por Brackett WW y col., ${ }^{27}$ quienes en este caso usaron premolares permanentes contralaterales libres de caries que iban a ser extraídas por motivos ortodóncicos. Bajo la misma metodología del trabajo pionero, un lado sirvió como grupo control, y el otro como grupo experimental aplicando la clorhexidina también al $2 \%$ después del grabado ácido y antes de un adhesivo convencional (Single Bond, 3M ESPE). Para el análisis en MET, algunos dientes fueron extraídos a los dos meses no observándose degradación en ninguno de los dos grupos, y en los dientes extraídos a los seis meses fue observada una ligera degradación del colágeno en el grupo control, mientras que en el grupo experimental no fue observada degradación.
Carrilho y col. ${ }^{28}$ relacionaron la degradación observada en la capa híbrida con la disminución de la resistencia de unión a la microtensión. Usaron terceros molares libres de caries divididos en grupos control (sin clorhexidina) y experimental (con clorhexidina), empleando el adhesivo Single Bond Plus (3M ESPE). Los dientes fueron extraídos algunos inmediatamente después de realizada la restauración, y el resto después de 14 meses de función intraoral. Se observó disminución de $35 \%$ en los valores de resistencia de unión en el grupo control después de los 14 meses en boca, mientras que en el grupo experimental no hubo reducción. Esto demostró la relación que existe entre las evidencias morfológicas de degradación de fibras colágenas en la capa híbrida y la reducción de la resistencia de unión con el tiempo, confirmando que la clorhexidina puede servir como inhibidor de dicho fenómeno. Brackett $\mathrm{MG}$ y col. ${ }^{29}$ usaron un adhesivo a base de acetona (Prime \& Bond NT, Dentsply/ Caulk) en premolares sanas contralaterales, dado que los tres estudios in vivo anteriores habían sido con adhesivos a base de etanol. Igualmente observaron en MET degradación extensa en los dientes del grupo control después de 12 meses, mientras que no observaron degradación en el grupo experimental. Recientemente Ricci y col. ${ }^{30}$ hicieron un estudio para comprobar la relación entre la aplicación de clorhexidina en dentina afectada por caries y disminución de la resistencia de unión. Los autores trabajaron con nińos de 8 a 11 ańos con molares deciduas cariadas contralaterales donde hicieron restauraciones de resina compuesta usando el adhesivo Prime \& Bond NT (Dentsply/Caulk). El periodo de función intraoral fue determinado por la exfoliación fisiológica de la pieza dentaria y los ensayos de microtensión determinaron que la mayor disminución en los valores de resistencia de unión en el grupo control fue dada a los 1-5 meses (30,6 \%), mientras que para el grupo experimental ocurrió a los 10-12 meses (26,3\%) demostrando de esta forma que el uso de clorhexidina puede retardar la degradación de las uniones resina-dentina.

\section{Discusión}

Hasta la fecha de envío de la presente revisión, son cinco los estudios in vivo disponibles en la literatura científica que incorporan una solución de clorhexidina al procedimiento clínico de aplicación de adhesivos convencionales 
obteniendo resultados positivos en la preservación de la capa híbrida, y de esa forma aumentando la longevidad de las uniones resina-dentina. ${ }^{15,27-30} \mathrm{El}$ protocolo usado en estos cinco estudios fue básicamente el mismo, el cual está sintetizado en el Cuadro 1. Con el objetivo de analizar y optimizar la forma como debería ser usada la clorhexidina han sido desarrollados diversos estudios in vitro que evalúan todas las variables que intervienen.

Moléculas de clorhexidina incluidas en la capa híbrida consiguen inhibir la actividad proteolítica de las MMPs, sin embargo la solución de clorhexidina puede ser aplicada en tres momentos diferentes: antes del grabado ácido, incorporado dentro del ácido fosfórico, o clorhexidina y diferentes tiempos de aplicación. Ellos concluyeron que el uso de clorhexidina a $0,002 \%$ durante 15 segundos parece ser suficiente para preservar las uniones resina-dentina durante un periodo de seis meses in vitro. Este bajo valor en la concentración mínima inhibitoria de las MMPs no difiere con los resultados del estudio de Gendron y col., ${ }^{14}$ quienes encontraron inhibición completa de las MMPs -2, -9 y -8 con concentraciones mínimas de clorhexidina a $0,0001 \%, 0,002 \%$ e $0,01 \%$ respectivamente. Por otro lado, la clorhexidina a $4 \%$ no consiguió preservar la resistencia de unión inmediata ni después de seis meses de almacenamiento en agua, con lo que podemos concluir que el rango de $0,002 \%$ a $2 \%$ de clorhexidina puede originar resulta-

Cuadro 1 PROTOCOLO DE APLICACIÓN DE ADHESIVOS CONVENCIONALES USANDO CLORHEXEDINA

Grabar la dentina con ácido fosfórico por 15 segundos y esmalte por 30 segundos

\begin{tabular}{l}
\hline Lavar con spray de agua y aire \\
\hline Retirar el exceso de humedad con papel absorbente o bolita de algodón esteriles \\
\hline Aplicar la solución de digluconato de clorhexidina al $2 \%$ de forma activa por 30 - 60 segundos \\
\hline Retirar el exceso de humedad con papel absorbente o bolita de algodón esteriles \\
\hline Aplicar el adhesivo convencional como manda el fabricante y fotoactivar \\
\hline continuar la restauración con resina compuesta
\end{tabular}

después del grabado ácido (esta última llamada de primer terapéutico y es la usada en los estudios in vivo). Ensayos in vitro con adhesivos convencionales mostraron que la resistencia de unión inmediata a dentina no es afectada cuando se usa clorhexidina antes o después del grabado ácido. ${ }^{21-23,31}$ Soares y col. $^{32}$ usaron clorhexidina antes, durante (incorporado en el ácido fosfórico) y después del grabado ácido en la cementación de restauraciones indirectas de resina compuesta, y no encontraron diferencia significante en la resistencia de unión inmediata. Stanislawczuc y col. ${ }^{33}$ hicieron un trabajo a largo plazo evaluando el efecto de la clorhexidina en la resistencia de unión inmediata y después de seis meses con dos adhesivos convencionales cuando era aplicada incorporada en el ácido fosfórico o como primer terapéutico. En ambos casos fue efectivo para reducir la degradación de las uniones resina-dentina después de seis meses de almacenamiento en agua sin mostrar diferencias significativas entre ambos grupos de tratamiento.

Con la finalidad de establecer la concentración mínima y máxima de clorhexidina que puede ser usada como primer terapéutico para la preservación de la resistencia de unión, Loguercio y col. ${ }^{34}$ evaluaron los efectos de diferentes concentraciones de digluconato de dos satisfactorios. La gran sustantividad de la clorhexidina, es decir, la capacidad de unirse a la matriz dentinaria para producir un mecanismo de liberación sostenida puede explicar el por qué de su acción prolongando la durabilidad de las uniones. ${ }^{35}$

Con respecto a las otras estrategias de unión, los estudios in vitro con adhesivos autograbadores son escasos y aún no otorgan una posición sólida respecto a la acción de la clorhexidina en la degradación de la capa híbrida. Tampoco existen trabajos in vivo que usen clorhexidina junto con adhesivos autograbadores y/o materiales autoadhesivos.

\section{Conclusión}

La incorporación de clorhexidina dentro del protocolo de aplicación de adhesivos convencionales es un recurso clínico válido para retardar la degradación de las fibras colágenas de la capa híbrida aumentando así la longevidad de las uniones resina-dentina.

\section{Referencias biblográficas}

1. Tyas MJ, Anusavice KJ, Frencken JE, Mount GJ. Minimal intervention dentistry--a review. FDI Com- mission Project 1-97. Int Dent J. 2000;50(1):1-12.

2. Phillips RW. Changing trends of dental restorative materials. Dent Clin North Am. 1989;33(2):28591.

3. Stanford JW, Weigel KV, Paffenbarger GC, Sweeney WT. Compressive properties of hard tooth tissues and some restorative materials. J Am Dent Assoc. 1960;60:746-56.

4. Kugel G, Ferrari M. The science of bonding: from first to sixth generation. J Am Dent Assoc. 2000;131 Suppl:20S-25S.

5. Van Meerbeek B, Vargas M, Inoue $S$, Yoshida Y, Peumans M, Lambrechts $\mathrm{P}$, et al. Adhesives and cements to promote preservation dentistry. Oper Dent. 2001;26(Suppl 6):11944

6. Van Meerbeek B, Peumans $M$, Poitevin A, Mine A, Van Ende A, Neves A, et al. Relationship between bond-strength tests and clinical outcomes. Dent Mater. 2010;26(2):e100-21.

7. Nakabayashi N, Kojima K, Masuhara $\mathrm{E}$. The promotion of adhesion by the infiltration of monomers into tooth substrates. J Biomed Mater Res. 1982;16(3):265-73.

8. Sano H, Shono T, Takatsu T, Hosoda $\mathrm{H}$. Microporous dentin zone beneath resin-impregnated layer. Oper Dent. 1994;19(2):59-64.

9. Hashimoto M, Ohno H, Kaga M, Endo K, Sano H, Oguchi H. In vivo degradation of resin-dentin bonds in humans over 1 to 3 years. J Dent Res. 2000;79(6):1385-91.

10. Breschi L, Mazzoni A, Ruggeri A, Cadenaro M, Di Lenarda R, De Stefano Dorigo E. Dental adhesion review: aging and stability of the bonded interface. Dent Mater. 2008;24(1):90-101.

11. Pashley DH, Tay FR, Yiu C, Hashimoto M, Breschi L, Carvalho RM, et al. Collagen degradation by hostderived enzymes during aging. J Dent Res. 2004;83(3):216-21.

12. Hannas AR, Pereira JC, Granjeiro JM, Tjäderhane L. The role of matrix metalloproteinases in the oral environment. Acta Odontol Scand. 2007;65(1):1-13.

13. Davies GE, Francis J, Martin AR, Rose FL, Swain G. 1:6-Di-4'-chlorophenyldiguanidohexane (hibita- 
ne); laboratory investigation of a new antibacterial agent of high potency. Br J Pharmacol Chemother. 1954;9(2):192-6.

14. Gendron R, Grenier D, Sorsa T, Mayrand D. Inhibition of the activities of matrix metalloproteinases 2, 8, and 9 by chlorhexidine. Clin Diagn Lab Immunol. 1999;6(3):437-9.

15. Hebling J, Pashley DH, Tjäderhane L, Tay FR. Chlorhexidine arrests subclinical degradation of dentin hybrid layers in vivo. J Dent Res. 2005;84(8):741-6. Erratum in: J Dent Res. 2006;85(4):384.

16. Lim KS, Kam PC. Chlorhexidine-pharmacology and clinical applications. Anaesth Intensive Care. 2008;36(4):502-12.

17. Brannstrom $M$. The hydrodynamic theory of dentinal pain: sensation in preparations, caries, and the dentinal crack syndrome. J Endod. 1986;12(10):453-7.

18. Meiers JC, Shook LW. Effect of disinfectants on the bond strength of composite to dentin. Am J Dent. 1996;9(1):11-4.

19. Meiers JC, Kresin JC. Cavity disinfectants and dentin bonding. Oper Dent. 1996;21(4):153-9.

20. Gürgan S, Bolay S, Kiremitçi A. Effect of disinfectant application methods on the bond strength of composite to dentin. J Oral Rehabil. 1999;26(10):836-40.

21. Perdigao J, Denehy GE, Swift EJ Jr. Effects of chlorhexidine on dentin surfaces and shear bond strengths. Am J Dent. 1994;7(2):81-4.

22. Bocangel JS, Kraul AOE, Vargas AG, Demarco FF, Matson E. Influence of disinfectant solutions on the tensile bond strength of a fourth generation dentin bonding agent. Pesq Odont Bras. 2000;14(2):10711.

23. De Castro FL, de Andrade MF, Duarte Júnior SL, Vaz LG, Ahid FJ. Effect of 2\% chlorhexidine on microtensile bond strength of composite to dentin. J Adhes Dent. 2003;5(2):129-38.

24. Van Noort R, Cardew GE, Howard IC, Noroozi S. The effect of local interfacial geometry on the measurement of the tensile bond strength to dentin. J Dent Res. 1991;70(5):889-93.

25. Vaidyanathan M, Sheehy EC, Gilbert SC, Beighton D. Antimicrobial properties of dentine bonding agents determined using in vitro and ex vivo methods. J Dent. 2009;37(7):514-21.

26. Tjäderhane L, Larjava $H$, Sorsa $T$, Uitto VJ, Larmas M, Salo T. The activation and function of host matrix metalloproteinases in dentin matrix breakdown in caries lesions. J Dent Res. 1998;77(8):1622-9.

27. Brackett WW, Tay FR, Brackett MG, Dib A, Sword RJ, Pashley $\mathrm{DH}$. The effect of chlorhexidine on dentin hybrid layers in vivo. Oper Dent. 2007;32(2):107-11.

28. Carrilho MR, Geraldeli S, Tay F, de Goes MF, Carvalho RM, Tjäderhane $\mathrm{L}$, et al. In vivo preservation of the hybrid layer by chlorhexidine. J Dent Res. 2007;86(6):529-33.

29. Brackett MG, Tay FR, Brackett WW, Dib A, Dipp FA, Mai S, et al. In vivo chlorhexidine stabilization of hybrid layers of an acetonebased dentin adhesive. Oper Dent. 2009;34(4):379-83.

30. Ricci HA, Sanabe ME, de Souza Costa CA, Pashley DH, Hebling J. Chlorhexidine increases the longevity of in vivo resin-dentin bonds. Eur J Oral Sci. 2010;118(4):411-6.

31. Campos EA, Correr GM, Leonardi DP, Pizzatto E, Morais EC. Influence of chlorhexidine concentration on microtensile bond strength of contemporary adhesive systems. Braz Oral Res. 2009;23(3):340-5.

32. Soares CJ, Pereira CA, Pereira JC, Santana FR, do Prado CJ. Effect of chlorhexidine application on microtensile bond strength to dentin. Oper Dent. 2008;33(2):183-8.

33. Stanislawczuk R, Amaral RC, Zander-Grande C, Gagler D, Reis A, Loguercio AD. Chlorhexidine-containing acid conditioner preserves the longevity of resin-dentin bonds. Oper Dent. 2009;34(4):481-90.

34. Loguercio AD, Stanislawczuk R, Polli LG, Costa JA, Michel MD, Reis A. Influence of chlorhexidine digluconate concentration and application time on resin-dentin bond strength durability. Eur J Oral Sci. 2009;117(5):587-96.

35.Carrilho MR, Carvalho RM, Sousa EN, Nicolau J, Breschi L, Mazzoni A, et al. Substantivity of chlorhexidine to human dentin. Dent Mater. 2010;26(8):779-85. 